\title{
Author Index to Volume 8
}

Bakker KA 161

Biddulph M 1, 5

Brand P 95

Brindley T 53

Comrie H 249

Comrie H 103

Franklin BJ 21
Goodey B 85, 99

Le Roux SW 161

Lipman A 135

Lloyd R 105

Low I 223

Nicks SC 179

Nomico M 207
Sanders P 207

Schaug E 147

Southworth B 119

Tait M 37

Thompson-Fawcett M 67

Watson D 97

White G 249

Young GA 161

\section{Keyword Index to Volume 8}

Action research 161

African City 207

Apartheid 179

Birmingham

Heartlands 21

Bordesley 21

Brazil 85

Cape Town - South Africa 223

Community 5, 37, 53

Community consultation 147

Community involvement 85

Community participation 135

Decentralisation 207

Developing environment 161

Discovery site 85
Economic apartheid 207 Local government 119

Fortified enclaves 207

Fragmentation re-created 207

Fragmented city 207

Humanist 'world view' 105

Imagination 223

Implementation 85

Informal trading 147

Inner city regeneration 161

Integration 179

Interface 179

Interpretation 85

Interpretive planning 85

Legible public realms 207

Lived space 67

Local activity 37
Local regionalism 135

Neighbourhood 5

New urbanism 67

Permeability 147

Porto Seguro 85

Post-apartheid 223

Poundbury 67

Practice 119

Public space 119

Reflective planning 67

Regeneration 5, 21

Robustness 147

Social change 53

Social sustainability 53

South Africa 119

South African

townships 147

Space 105
Spatial imagination 105

Spatial

transformation 223

Sustainable urban

development 53

Synthesis 105

Theory 119

Traditional 135

Transformation 161, 207

Understandings of heritage 85

Upgrading 179

Urban migration/

exodus 207

Urban

(re)development 207

Urban renewal 223

Urban/rural dichotomy 135

Urban

village $21,37,53,67$ 